\title{
Collisions of relativistic clusters and the formation of black holes
}

\author{
Stuart L. Shapiro and Saul A. Teukolsky \\ Center for Radiophysics and Space Research and Departments of Astronomy and Physics, \\ Cornell University, Ithaca, New York 14853
}

(Received 30 October 1991)

\begin{abstract}
We perform numerical simulations of head-on collisions of relativistic clusters. The cluster particles interact only gravitationally, and so satisfy the collisionless Boltzmann equation in general relativity. We construct and follow the evolution of three classes of initial configurations: spheres of particles at rest; spheres of particles boosted towards each other; and spheres of particles in circular orbits about their respective centers. In the first two cases, the spheres implode towards their centers and may form black holes before colliding. These scenarios thus can be used to study the head-on collision of two black holes. In the third case the clusters are initially in equilibrium and cannot implode. In this case collision from rest leads either to coalescence and virialization, or collapse to a black hole. This scenario is the collisionless analog of colliding neutron stars in relativistic hydrodynamics.
\end{abstract}

PACS number(s): 04.20.Jb, 95.30.Sf, 97.60.Lf

\section{INTRODUCTION}

A time-honored means of probing the nature of an interaction in physics is to perform collision experiments. Now, by means of numerical simulations, it is possible to perform such experiments to study the gravitational interaction. In addition to gaining theoretical understanding from these simulations, one can model strong-field encounters that may actually occur in nature. Examples include collisions between neutron stars and between black holes.

In this paper we explore collisions between relativistic spheres of particles according to general relativity. The particles are assumed to interact exclusively by gravitation, i.e., they obey the relativistic collisionless Boltzmann equation (Vlasov equation). For this purpose, we have constructed a numerical code that solves Einstein's equations for the gravitational field coupled to matter sources obeying the Vlasov equation. This is the same mean-field, particle simulation code described in Refs. [1] and [2] to study nonspherical gravitational collapse. The code is designed to handle axisymmetric systems with no net angular momentum. The present version assumes equatorial symmetry. It is thus suitable for studying head-on collisions between identical nonrotating clusters of particles.

We consider three different collision scenarios: (1) the collision from rest of spheres of particles with no initial internal motion; (2) the collision of the same spheres initially boosted towards each other with a uniform velocity; (3) the collision from rest of equilibrium spheres of particles, i.e., spheres in which the internal particle motions maintain dynamical equilibrium when the spheres are widely separated.

In the first two cases the nonequilibrium spheres collapse towards their own centers and may form black holes before they collide. Thus these scenarios provide a way of studying the head-on collision of two identical nonrotating black holes. This treatment is an alternative means of analyzing this important problem, which has only been considered [3] previously using the vacuum Einstein equations ("topological" black holes). We thus demonstrate how a code designed to handle matter in general relativity can be used to treat black holes, without having to develop special-purpose routines to handle the boundary conditions for vacuum black holes.

The third case is the cluster analogue of the head-on collision of two neutron stars [4]. Collisions of equilibrium clusters have previously been studied only in Newtonian gravitation [5]. In a strong gravitational field the collision can lead to black-hole formation. However, stable equilibrium clusters do not implode on their own centers. Thus a black hole can only form when the clusters interact closely.

For each of these three cases, we first construct solutions of the initial-value equations of general relativity. We describe these solutions in some detail because they may be useful for other simulations. For example, case (2) furnishes a non-time-symmetric solution of the initial-value equations with matter. Most previous investigations of these equations assume time symmetry because it is analytically simpler.

\section{INITIAL DATA}

To follow the head-on collision of two clusters, we solve the field equations in $3+1$ form following Arnowitt, Deser, and Misner (ADM) [6]. We use maximal time slicing and isotropic spatial coordinates in axisymmetry. The metric is

$$
\begin{aligned}
d s^{2}= & -\alpha^{2} d t^{2}+A^{2}\left(d r+\beta^{r} d t\right)^{2} \\
& +A^{2} r^{2}\left(d \theta+\beta^{\theta} d t\right)^{2}+B^{2} r^{2} \sin ^{2} \theta d \phi^{2} .
\end{aligned}
$$

The matter satisfies the relativistic Vlasov equation, which we solve by particle simulation in the mean gravitational field. The basic code is identical to the one described in Refs. [1] and [2]. We list the key equations in 
the Appendix.

The initial configurations are all generalizations of the momentarily static spheroids that were constructed in Ref. [7]. We adopt the same notation as in that paper.

The axisymmetric three-metric that we adopt at $t=0$ is the "radiation-free" conformally flat form

$$
{ }^{(3)} d s^{2}=\psi^{4} \delta_{i j} d x^{i} d x^{j} \text {. }
$$

Here we have chosen the "radiation variable" $\eta=\ln (A / B)$ to be zero at $t=0$, and hence

$$
A=B=\psi^{2} .
$$

For a moment of time symmetry, the shifts $\beta^{i}$ and all components $K^{i}{ }_{j}$ of the extrinsic curvature are zero. The only Einstein equation that the initial data must satisfy nontrivially is the Hamiltonian constraint

$$
{ }^{(3)} R=16 \pi \rho^{*},
$$

where $\rho^{*}=T_{\mu \nu} n^{\mu} n^{\nu}$ and $n^{\mu}$ is the normal vector to the initial hypersurface $t=0$. Here ${ }^{(3)} R$ is the Ricci scalar and $T_{\mu \nu}$ is the stress-energy tensor for a gas of collisionless particles. The Hamiltonian constraint reduces to

$$
\nabla^{2} \psi=-2 \pi \psi^{5} \rho^{*}
$$

with the boundary conditions

$$
\nabla \psi=0, \quad r=0, \quad \psi \rightarrow 1+\frac{M}{2 r}, \quad r \rightarrow \infty,
$$

where $M$ is the total mass-energy of the configuration.

Consider two spheres of particles of radius $a$ separated by a coordinate distance $2 z_{0}$ along the $z$ axis (see Fig. 1). In Newtonian theory, if the spheres are homogeneous and each of mass $M_{N} / 2$, then the density is

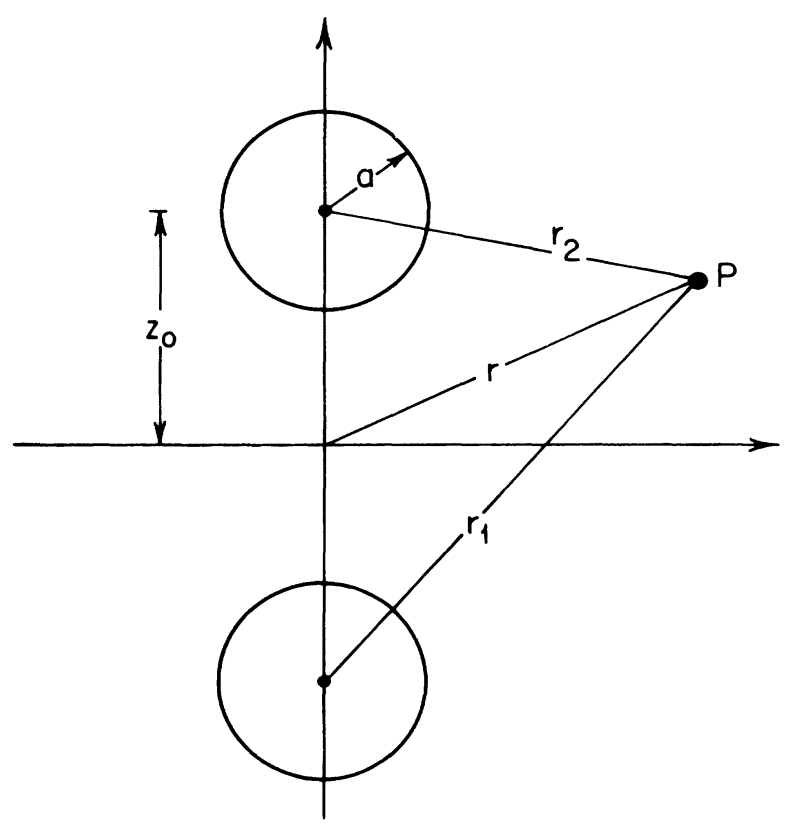
cles.

$$
\rho_{N}=\frac{M_{N} / 2}{4 \pi a^{3} / 3}
$$

everywhere inside the spheres. The Newtonian gravitational potential $\Phi_{N}$ satisfies Poisson's equation

$$
\nabla^{2} \Phi_{N}=4 \pi \rho_{N}
$$

In the lower half-plane, the solution is

$$
\Phi_{N}=\left\{\begin{array}{l}
-\frac{M_{N}}{2 r_{1}}-\frac{M_{N}}{2 r_{2}}, \quad r_{1} \geq a, \\
-\frac{M_{N}}{4 a}\left(3-\frac{r_{1}^{2}}{a^{2}}\right)-\frac{M_{N}}{2 r_{2}}, \quad r_{1}<a,
\end{array}\right.
$$

where

$$
\begin{aligned}
& r_{1}=\left[x^{2}+y^{2}+\left(z+z_{0}\right)^{2}\right]^{1 / 2}, \\
& r_{2}=\left[x^{2}+y^{2}+\left(z-z_{0}\right)^{2}\right]^{1 / 2} .
\end{aligned}
$$

The solution in the upper half-plane is obtained by interchanging $r_{1}$ and $r_{2}$.

Now to obtain an analytic solution to the Hamiltonian constraint (2.5), set the density profile $\rho^{*}$ by

$$
2 \pi \psi^{5} \rho^{*} \equiv 4 \pi \rho_{N}
$$

Comparing the Poisson's Eq. (2.8), we immediately conclude that

$$
\psi=1-\Phi_{N}
$$

Since at large radii $\Phi_{N} \rightarrow-M_{N} / r$, we have, from Eqs. (2.6) and (2.12),

$$
M=2 M_{N} \text {. }
$$

Note that outside the matter the three-metric is the same as the solution given by Misner and Wheeler [8] for two time-symmetric black holes. When the spheres are far apart, the initial data describes two spherical black holes at rest whenever $a<0.25 M$ in the adopted isotropic coordinates.

The total rest mass of the configuration is

$$
M_{0}=\int \frac{\rho^{*}}{\gamma} \psi^{6} d^{3} x
$$

where, for a moment of time symmetry,

$$
\gamma=-u^{\mu} n_{\mu}
$$

and $u^{\mu}$ is the particle four-velocity. The total rest mass is related to the total number of particles $N$ by

$$
M_{0}=m N \text {, }
$$

where $m$ is the rest mass of a particle. In the special case that the particles are initially at rest, $\gamma=1$ and

$$
M_{0}=\int 2 \rho_{N}\left(1-\Phi_{N}\right) d^{3} x=2 M_{N}+4 W_{N},
$$

where

$$
W_{N}=-\frac{1}{2} \int \rho_{N} \phi_{N} d^{3} x
$$


is the Newtonian gravitational binding energy. When the spheres do not overlap,

$$
W_{N}=\frac{M_{N}^{2}}{a}\left[\frac{3}{10}+\frac{1}{8} \frac{a}{z_{0}}\right] .
$$

Having determined the density profile, we now must specify a velocity profile. Once it is given, we can then distribute particles to sample the initial phase-space distribution function. We consider three different collision scenarios, corresponding to three different initial velocity profiles: spheres of particles initially at rest, spheres of particles boosted towards each other with a $z$ velocity, and spheres of particles in circular orbits about their respective centers. The initial data for the first and third cases are at a moment of time symmetry, for which the equations given above apply. In the third case we choose the circular velocity profile so that the spheres would be in dynamical equilibrium if they were very far apart. Case (2) does not correspond to a moment of time symmetry. The equations for this case will be discussed below.

\section{A. Spheres of particles at rest}

In this case, each particle is initially at rest and hence is assigned initial four-velocity components

$$
u_{r}=0, u_{\theta}=0, u_{\phi}=0 \text {. }
$$

The time component of the four-velocity is determined by the normalization condition $u^{\mu} u_{\mu}=-1$ [cf. Eq. (A16)]. From Eqs. (2.13) and (2.16)-(2.19), the rest mass $m$ of a particle can be determined once the total number of particles $N$ in the cluster is chosen. By the equivalence principle, the dynamical behavior of the collisionless gas is independent of $N$. Of course, $N$ must be sufficiently large in a numerical simulation that statistical fluctuations are unimportant.

Equation (2.14) can now be employed to distribute particles in the interior of the spheres. We have

$$
N=\frac{M_{0}}{m}=\frac{1}{m} \int \rho^{*} \psi^{6} d^{3} x=\frac{3}{2} \frac{M_{N}}{m a^{3}} \int \psi d\left(\frac{r^{3}}{3}\right) d(\cos \theta),
$$

where we have used Eqs. (2.11) and (2.7) to express $\rho^{*}$ in terms of $\rho_{N}$. The integrand in Eq. (2.21) gives the particle distribution in spherical polar coordinates $r$ and $\theta$. Sampling from this distribution by a rejection method, we can assign $(r, \theta)$ coordinates to the particles. By axisymmetry, the particles are uniformly distributed in $\phi$.

This assignment of particle positions and velocities, together with the analytic three-metric, completely solves the initial-value problem. The configuration consists of two identical spheres of the type constructed in Ref. [7], initially at rest and separated by a coordinate distance $2 z_{0}$ along the axis. In the Newtonian limit the spheres are homogeneous, but relativistic configurations are inhomogeneous with density increasing outwards.

\section{B. Boosted spheres}

We next construct initial data which corresponds to the same two spheres described above, but boosted towards each other with velocity $v$ along the $z$ axis. To completely specify the boost, we must designate which set of observers measures the velocity $v$. We choose the normal observers. While arbitrary, this choice does eliminate dependence of the velocity on the shift vector. The particle coordinate positions are assigned exactly as in the previous case. The coordinate components of the particle four-velocities are

$u_{r}=A \gamma v \cos \theta, u_{\theta}=-A r \gamma v \sin \theta, u_{\phi}=0$,

where

$$
\gamma=\left(1-v^{2}\right)^{-1 / 2}
$$

With this assignment of phase-space particle coordinates, the configuration is no longer at a moment of time symmetry at $t=0$. We are still free to set the dynamical variables $\eta$ and $K_{\theta}^{r}$ to zero. However, the full set of momentum and Hamiltonian constraint equations has to be solved. We proceed by iteration: first we calculate $A$ and $m$ analytically for $v=0$ and total mass $M$ as in the previous case. The particle positions, together with the velocities of Eq. (2.22), then allow us to calculate the matter source terms (A17)-(A21) for the field equations. Next we solve the momentum constraints, Eqs. (A4) and (A5), the Hamiltonian constraint (A6), the lapse Eq. (A7), and the shift Eqs. (A8) and (A9). We then compute the mass from Eq. (A22). In general it will not be equal to $M$ because we have obviously added energy by boosting the spheres. We then adjust the particle mass $m$ and, using the updated values of $A$, we recalculate the source terms. We solve again the field equations and get a new value of $M$. The process is repeated until convergence is achieved. The result is a self-consistent solution of the initial-value equations with matter but without time symmetry.

Our choice to set $\eta$ and $K^{r}{ }_{\theta}$ to zero is computationally convenient, but arbitrary. The choice is also motivated by the desire to minimize the amount of gravitational radiation put into the initial data. An alternative choice would have been to set the transverse part of the extrinsic curvature to zero, and solve for the longitudinal part. Since the dominant burst of gravitational radiation arises from the collision and coalescence of the two spheres, we have ignored small differences in the choices of the initial data and opted for the simplest numerical prescription.

\section{Equilibrium spheres}

The spheres constructed in the previous two cases, when widely separated, each implode to black holes because they are not in dynamical equilibrium. In particular, they do not have any centrifugal support to hold back their inward gravitational collapse. Now we wish to construct spheres of particles which, when widely separated, are in dynamical equilibrium. We do this by arranging all the particles to be in randomly oriented circular orbits with centrifugal forces exactly balancing gravity. Each of these clusters is thus an Einstein [9] cluster in the absence 
of the companion. We choose the particle distribution so that the density $\rho^{*}$ is again given by Eq. (2.11). Because the spheres are at rest at $t=0$, the initial data corresponds to a moment of time symmetry, and the Hamiltonian constraint is the only nontrivial initial-value equation. The Hamiltonian constraint once again can be solved analytically, and $\psi$ is again given by Eqs. (2.12) and (2.9).

The magnitude of the equilibrium velocity $v_{\perp}$ as measured by a normal observer is given by [10]

$v_{\perp}=\left(\frac{m\left(r_{S}\right)}{r_{S}}\right)^{1 / 2}\left(1-\frac{2 m\left(r_{S}\right)}{r_{S}}\right)^{-1 / 2}=\left(\frac{g_{r r}^{S}-1}{2}\right)^{1 / 2}$.

Here $r_{S}$ is the Schwarzschild radial coordinate measured from the center of the sphere, $m\left(r_{S}\right)$ is the Schwarzschild mass inside radius $r_{S}$, and $g_{r r}^{S}$ is the Schwarzschild radial metric coefficient. Transforming to isotropic coordinates, and using the explicit form of the metric in Eqs. (2.12) and (2.9), we obtain

$v_{1}=\left[\frac{M_{N} r_{1}^{2}}{a^{3}}\right]^{1 / 2} \frac{\left[1+\left(3 M_{N} / 4 a\right)\left(1-r_{1}^{2} / a^{2}\right)\right]^{1 / 2}}{1+\left(M_{N} / 4 a\right)\left(3-5 r_{1}^{2} / a^{2}\right)}$.

Particle positions can now be assigned by rejection similarly to the case of particles at rest. The only difference is that there is a factor of $1 / \gamma$ in the integrand of Eq. (2.21) because of the circular motion. Here

$$
\gamma=\left(1-v_{\perp}^{2}\right)^{-1 / 2}
$$

The components of the three-velocity of a particle in the orthonomal frame of a normal observer are

$v^{\widehat{r}}=\left(\frac{z_{0} \sin \theta}{r_{1}}\right) v_{\perp} \sin \chi, \quad v^{\hat{\theta}}=\left(\frac{r+z_{0} \cos \theta}{r_{1}}\right) v_{\perp} \sin \chi$,

$v^{\hat{\phi}}=v_{\perp} \cos \chi$.

Here $\chi$ is an angle chosen from a uniform distribution in $[0,2 \pi]$. The corresponding four-velocity components are

$u_{r}=A \gamma v^{\hat{p}}, \quad u_{\theta}=A r \gamma v^{\hat{\theta}}, u_{\phi}=A r \sin \theta \gamma v^{\hat{\phi}}$.

This prescription for the initial fields and matter sources provides another exact solution of the initial value equations. When the spheres are widely separated $\left(a / z_{0} \rightarrow 0\right)$, the configuration corresponds to two identical spheres in equilibrium that will collide head-on from rest.

\section{GEOMETRIC PROBES}

We construct a number of geometric diagnostics to probe the spacetime during a simulation. We monitor the conservation of mass-energy by calculating the Brill mass at regular time intervals [see Eq. (A22)]. We search for the presence of black holes by locating apparent horizons, and compute their shape and area when they are present. We probe for both a common horizon enclosing both clusters (one black hole), and disjoint horizons surrounding each of them separately (two black holes).

Starting, for example, from Eq. (29) of Ref. [11], we derive parameteric equations for an apparent horizon $r=r(s), \theta=\theta(s)$ in the form

$$
\begin{aligned}
& \frac{d^{2} r}{d s^{2}}=-\frac{A_{, r}}{A}\left[\frac{d r}{d s}\right]^{2}-\left[2 \frac{A_{, \theta}}{A}+\frac{B_{, \theta}}{B}+\cot \theta\right) \frac{d r}{d s} \frac{d \theta}{d s}+\left[r^{2} \frac{A_{, r}}{A}+r^{2} \frac{B_{, r}}{B}+2 r\right)\left(\frac{d \theta}{d s}\right]^{2}-r \mathcal{N} \frac{d \theta}{d s}, \\
& \left.\left.\frac{d^{2} \theta}{d s^{2}}=\frac{1}{r^{2}}\left(\frac{A_{, \theta}}{A}+\frac{B_{, \theta}}{B}+\cot \theta\right)\left(\frac{d r}{d s}\right)^{2}-\left(\frac{2 A_{, r}}{A}+\frac{B_{, r}}{B}+\frac{3}{r}\right) \frac{d r}{d s} \frac{d \theta}{d s}-\frac{A_{, \theta}}{A}\right) \frac{d \theta}{d s}\right]^{2}+\frac{1}{r} \mathcal{N} \frac{d r}{d s},
\end{aligned}
$$

where $s$ is the arc length and

$$
\mathcal{N} \equiv\left(\frac{d r}{d s}\right)^{2} \frac{1}{B} \hat{K}_{r}^{r}+2 \frac{d r}{d s} \frac{d \theta}{d s} \frac{1}{B} \hat{K}_{\theta}^{r}-\left(\frac{d \theta}{d s}\right)^{2} \frac{r^{2}}{B}\left(\hat{K}_{r}^{r}+\hat{K}_{\phi}^{\phi}\right)+\frac{1}{A^{2} B} \hat{K}_{\phi}^{\phi} .
$$

These equations must be solved subject to the initial conditions

$$
s=0: \quad r=r_{0}, \theta=0, \frac{d r}{d s}=0, \frac{d \theta}{d s}=\frac{1}{A r} .
$$

Note that Eq. (3.1) is indeterminate at $s=0$. L'Hôpital's rule gives

$$
\frac{d^{2} r}{d s^{2}}=\frac{1}{2}\left(\frac{A_{, r}}{A}+\frac{B_{, r}}{B}+\frac{2}{r}\right) \frac{1}{A^{2}}-\frac{1}{A^{3} B} \hat{K}_{\phi}^{\phi}(s=0) \text {. }
$$

A common horizon is a solution of Eqs. (3.1)-(3.4) that also satisfies the condition $d r / d s=0$ at $\theta=\pi$. Equatorial symmetry allows us instead to satisfy the condition $d r / d s=0$ at $\theta=\pi / 2$. The general strategy we adopt for finding a common horizon is to start at the pole with a trial value $r=r_{0}$ and integrate Eqs. (3.1) and (3.2) to the equator. Vary $r_{0}$ and search for a sign change of $d r / d s$ at the equator. If there is no sign change for any $r_{0}$, there is no common horizon. If there is a sign change, iterate until $d r / d s=0$ to locate $r_{0}$ precisely.

A disjoint horizon in the upper hemisphere is a solu- 
tion of Eqs. (3.1) - (3.4) for which $\theta$ not only starts at zero, but also returns to zero in the same hemisphere with $d r / d s=0$. By equatorial symmetry there is an identical horizon in the lower hemisphere. An iterative procedure similar to the case of a common horizon can be used to search for a disjoint horizon [12].

As in Ref. [7], we compute the area and circumferences of any apparent horizon that is found. The area is

$$
\mathcal{A}=2 \pi \int_{0}^{s_{\max }} B r \sin \theta d s
$$

where $s_{\max }$ is the terminal value of $s$. The polar circumference of an apparent horizon is

$$
e_{\text {pole }}=2 s_{\max } \text {, }
$$

while the equatorial circumference of a common horizon is

$$
\mathcal{C}_{\mathrm{eq}}=\left.2 \pi B r\right|_{\theta=\pi / 2} \text {. }
$$

\section{RESULTS}

The basic code employed to solve the equations listed in the Appendix was described briefly in Refs. [1] and [2]. We use similar computational resources to those reported there, typically 100 radial and 32 angular zones, and 6000 test particles. Again, a key feature enabling us to track motion approaching the origin or close to the axis is that the angular grid can fan and the radial grid can contract to follow the matter.

\section{A. Spheres of particles at rest}

To explore the range of possible initial data, we considered spheres with a variety of radii and separations. Three interesting choices, all involving black holes, are shown in Fig. 2. When the spheres are widely separated $\left(z_{0} \gg M\right)$, they are black holes if $a<0.25 M$. When $z_{0} \sim M$, the critical radius is somewhat less. Figure 2(a) shows one of the spheres in the upper half-plane with $a=0.1 M$ and $z_{0}=0.4 M$. The spheres are too far apart for there to be a common horizon, but there is a disjoint horizon. From the location of the horizon, we see that any value of $a$ up to about $0.18 M$ would give the same horizon, since the exterior metric is independent of $a$. The area of a single Schwarzchild black hole of mass $M / 2$ is $4 \pi M^{2}$, which is a convenient unit for the area of disjoint horizons. Likewise we use the unit $2 \pi M$ for the circumference of a disjoint horizon. The area of the horizon in Fig. 2 (a) is $\mathcal{A} / 4 \pi M^{2}=1.72$. Its polar circumference is $\bigodot_{\text {pole }} / 2 \pi M=1.32$.

Because the collision results in the formation of a single Schwarzschild black hole, one can use the area theorem to provide an upper limit to the amount of gravitational radiation that can be emitted during this encounter:

$$
\frac{\Delta E_{\mathrm{rad}}}{M}=1-\frac{M_{\mathrm{final}}}{M} \leq 1-\left[\frac{\mathcal{A}}{8 \pi M^{2}}\right]^{1 / 2}=0.073 \text {. }
$$

Note that to guarantee that the radiated energy is posi- tive, the initial value of the area of a disjoint horizon $\mathcal{A} / 4 \pi M^{2}$ has to be less than two.

We used our dynamical code to evolve this initial data. We found that, as in the case of computations of gravitational radiation from colliding black holes in vacuum spacetimes [3], here also the actual emission is considerably less than the upper limit. In fact, for all the cases reported in this paper, the gravitational radiation efficiency

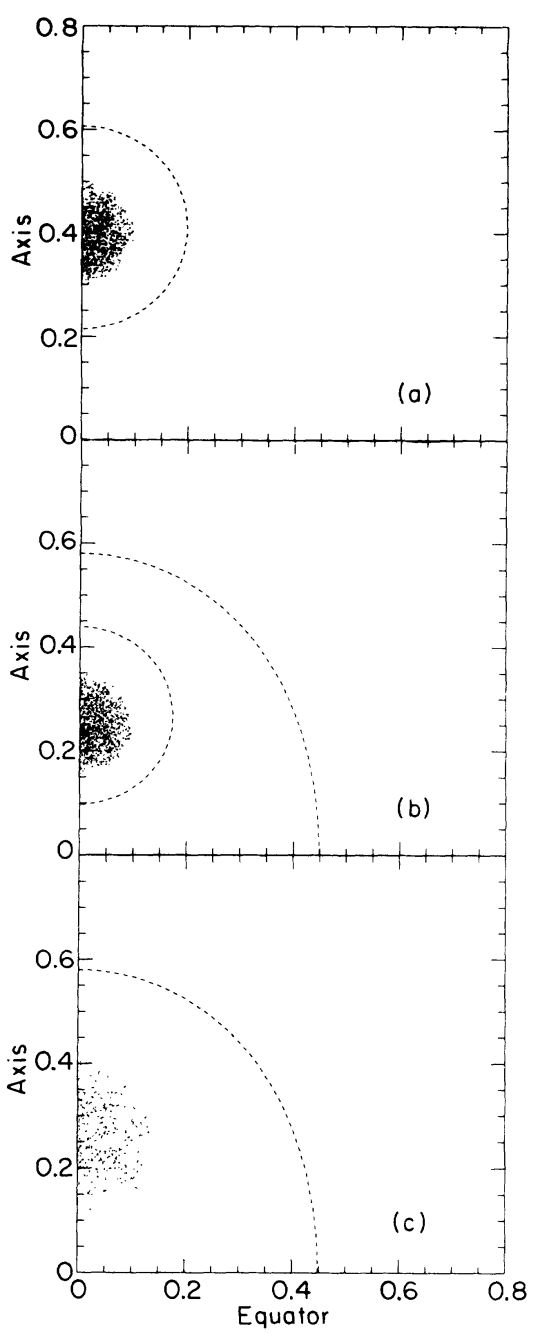

FIG. 2. Snapshots of particle positions at $t=0$ for spheres of particles initially at rest. The coordinate positions (in units of $M)$ are projected onto a meridional plane. Because of symmetry, we show only one quadrant in the upper hemisphere: there is an identical sphere in the lower hemisphere. Dashed lines show the locations of apparent horizons. The three cases shown describe three different initial data sets for black holes colliding from rest. In case (a) the spheres are sufficiently compact that they each are surrounded by a disjoint horizon, but they are far enough apart that there is no common horizon. In case (b) they have been moved close enough that now there is also a common horizon. In case (c) the radius of each sphere has been increased. Now there are no disjoint horizons, but only a common horizon. Numerical values of the initial parameters and horizon measures for each case are given in the text. 
is much less than $1 \%$ by the time we terminate the integrations.

Figure 2(b) shows the initial data for two spheres of the same radius as in Fig. 2(a), but separated by only $z_{0}=0.25 M$. Now there is also a common horizon. Normalizing to a Schwarzschild black hole of mass $M$, we find that its equatorial and polar circumferences are $\bigodot_{\text {eq }} / 4 \pi M=0.87, C_{\text {pole }} / 4 \pi M=1.06$. Despite its nonspherical shape, its area is $\mathcal{A} / 16 \pi M^{2}=1.00$. The vacuum geometry is identical to the case examined by Bishop [13], and we are in good agreement with the numerical values he reports for the various horizon parameters. Evolution of this data leads to a more spherical black hole, as expected.

In Fig. 2(c) the separation is the same as in Fig. 2(b), but the radius of the spheres has been increased to $0.16 M$. We see that the disjoint horizon has disappeared, while the common horizon has precisely the same structure as in Fig. 2(b).

\section{B. Boosted spheres}

Next we consider a sequence of spheres, "identical" to the ones considered in Sec. IV A, but now boosted towards each other by increasing amounts. The spheres each have a radius $a=0.8 M$ and are separated by $z_{0}=1.4 M$, so there are no apparent horizons initially. These nonequilibrium spheres implode on their own centers while approaching each other head-on.

Figure 3 shows the evolution of the first member of the sequence, where there is no velocity boost. At $t \simeq 5 M$ a disjoint horizon forms around each of the spheres. The spheres then implode rapidly toward their centers. We are forced to terminate the integrations when the field gradients near the cluster centers become so large that we can no longer maintain accuracy. This is the same problem that limits integrations of spherical collapse to black holes with maximal time slicing and isotropic coordinates [14]. By the time the lapse has fallen exponentially to $\sim 10^{-2}$ at the cluster center, the coordinate separation of the spheres is still $>1 M$. Thus these coordinates are not adequate to follow the entire collision and coalescence.

Figure 4 shows the evolution of spheres with an initial boost velocity $v=0.12$. Again the spheres implode, and a disjoint horizon appears at $t \simeq 6 M$. Now, however, the boost enables the clusters to get closer before the integrations are forced to terminate. By the end of the simulation a common horizon envelops the system.

In Fig. 5 we increase the boost velocity to $v=0.15$. This time a common horizon appears before the disjoint horizon. Finally, in Fig. 6 we show the case for $v=0.5$. A common horizon appears, but no disjoint horizon, and the two spheres actually make contact inside the black hole as the integrations terminate.

The sequence of boosted cases is a sequence of increasing kinetic energy. This is reflected in the values of the ratio $M / M_{0}$ along the sequence $0.68,0.74,0.76$, and 1.30 respectively. In the first three cases the velocity is low enough that negative gravitational binding energy dominates over the kinetic energy contribution to the total mass-energy $M$. In the last case, kinetic energy dom- inates and the encounter is hyperbolic. The case $v \sim 0.3$ would give $M / M_{0}=1$, which corresponds to free fall from rest at infinity.

We have numerical evidence that one cannot find initial data for this sequence with $v$ arbitrarily close to one. The iteration of the combined constraint and gauge equations described in Sec. II B fails to converge. The reason appears to be that $g_{00}$ changes sign, which implies that $t$ is no longer a timelike coordinate. Hence the adopted form of the ADM equations breaks down.

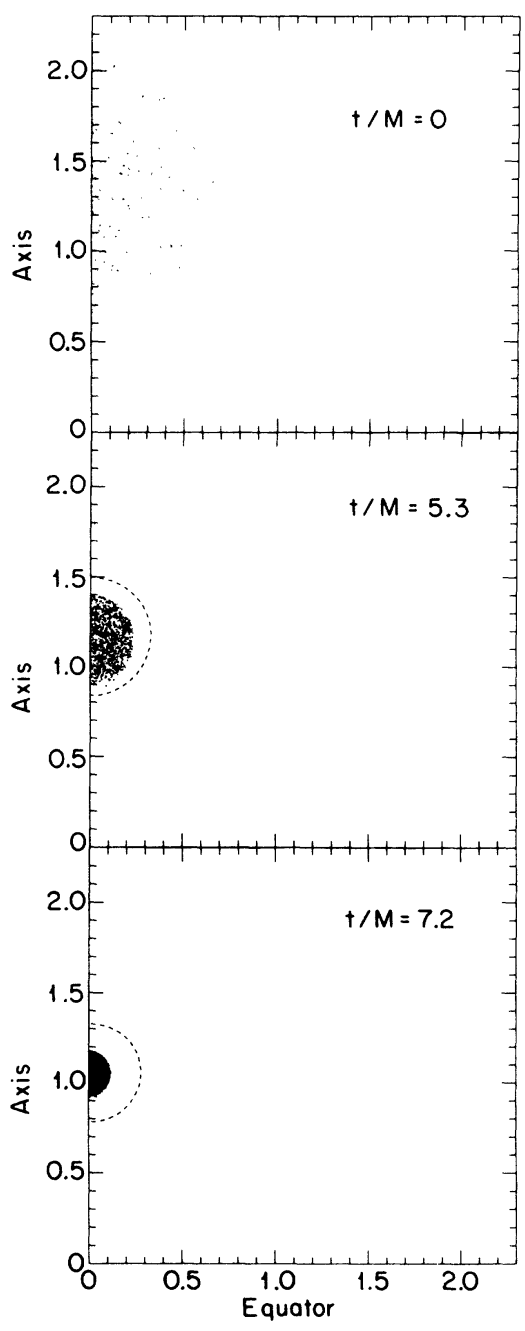

FIG. 3. Snapshots of particle positions at selected times for the collision of two spheres of particles initially at rest. The initial radius of each sphere is $a=0.8 M$, and their centers are at $z_{0}= \pm 1.4 M$. The spheres implode on their own centers as they fall toward each other, forming disjoint horizons at $t \simeq 5 M$. The area of each disjoint horizon is $\mathcal{A} / 4 \pi M^{2}=1.2$ at the end of the integration. While this value must be 1 if the black holes are far apart, it can be greater than 1 when they interact, but must always be less than 2. The polar circumference of the horizon is $e_{\text {pole }} / 2 \pi M=1.1$. Again the deviation from 1 is due to interactions. 


\section{Equilibrium spheres}

Working with equilibrium spheres allows us to study collisions without the clusters imploding on their own centers before making contact. This is the collisionless analogue of the head-on collision of neutron stars in hydrodynamics.

In Newtonian theory the head-on collision from rest of two clusters leads to violent relaxation, coalescence, and virialization to a new equilibrium state [5]. In general relativity, however, there is the possibility that the collision will be followed by collapse to a black hole. To anticipate when this might happen, note first that a single spherical

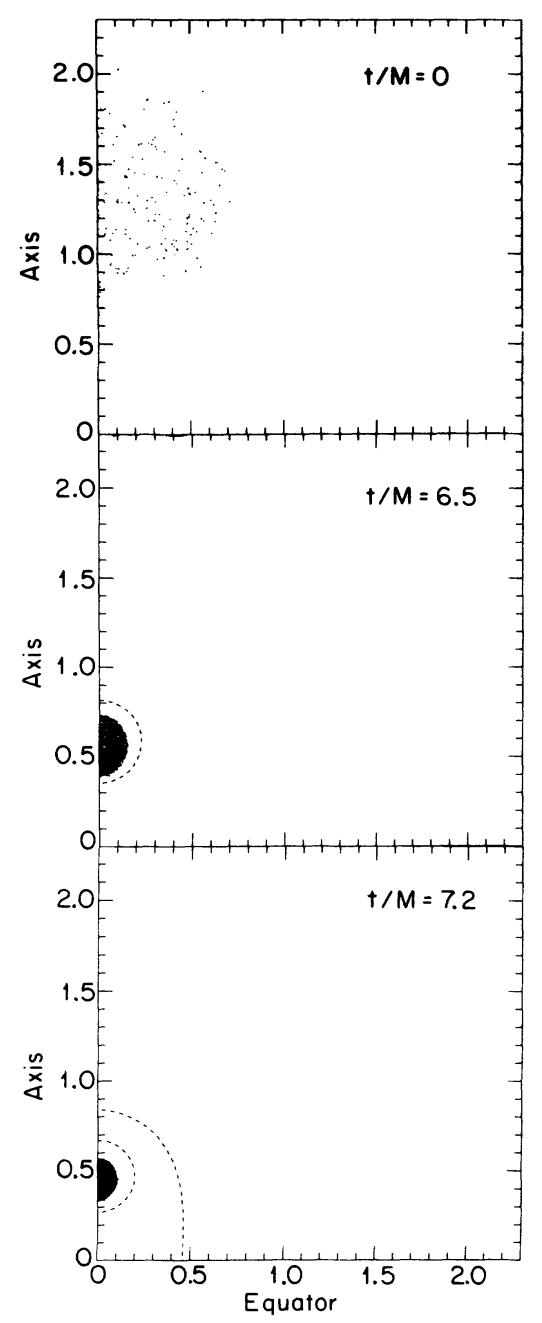

FIG. 4. Snapshots of particle positions at selected times for the collision of the same spheres shown in Fig. 3, but now boosted towards each other with a velocity $v=0.12$. Because of the boost, the clusters can get close enough for a common horizon to form as well as the disjoint horizons. The area of each disjoint horizon is $\mathcal{A} / 4 \pi M^{2}=1.1$ at the end of the integration, while the area of the common horizon is $\mathcal{A} / 16 \pi M^{2}=0.95$. The polar circumference of the disjoint horizon is $\mathcal{C}_{\text {pole }} / 2 \pi M=1.1$. The circumferences of the common horizon are $\mathcal{C}_{\text {eq }} / 4 \pi M=0.70$ and $e_{\text {pole }} / 4 \pi M=1.1$. cluster is dynamically unstable to collapse whenever its radius in Schwarzschild coordinates is less than $6 M$, corresponding to an isotropic radius of $4.95 M$. Numerical examples of this phenomenon are given in Ref. [10]. Two well-separated spheres of mass $M / 2$ are thus individually unstable if $a<2.47 M$. If these spheres were suddenly superposed, the resulting sphere would be unstable if the original $a$ was less than $4.95 M$. In fact, the final sphere would be unstable for even larger values of $a$ because the original internal velocities that maintained equilibrium were those appropriate to a mass $M / 2$ rather than $M$. We thus expect to find for a value of $a \gtrsim 5 M$ a transition between collisions leading to black-hole formation and those leading to virialization to a merged equilibrium.

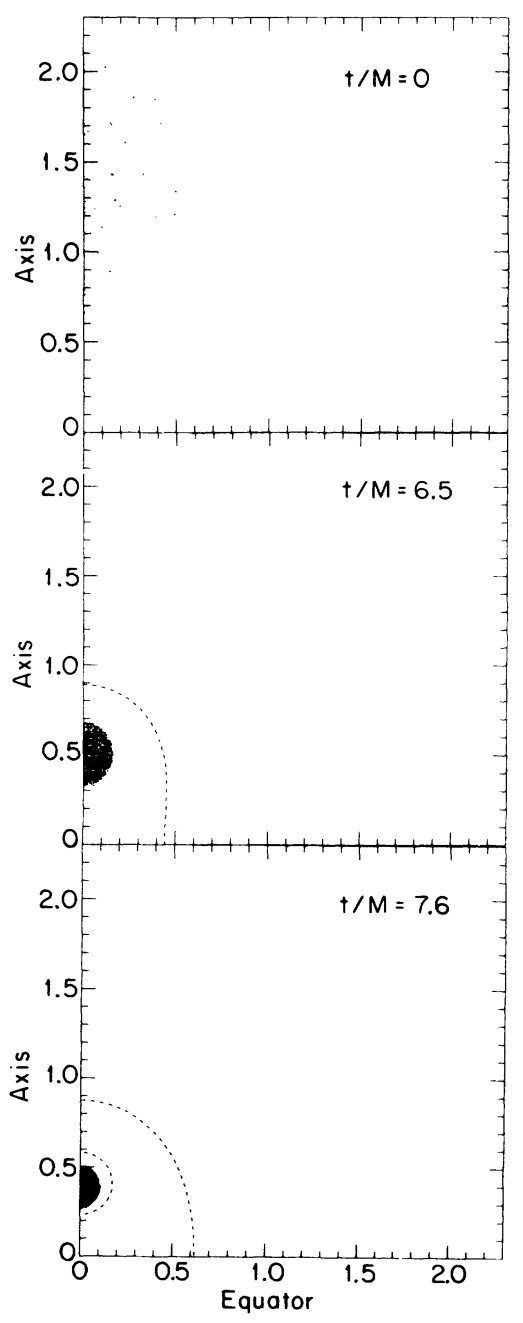

FIG. 5. Snapshots of particle positions at selected times for the collision of the same spheres shown in Fig. 3, but boosted towards each other with a velocity $v=0.15$. Now the common horizon appears first. At $t / M=6.5$ its area is $\mathcal{A} / 16 \pi M^{2}=0.86$, and it grows to 0.99 by the end of the integration, when its circumferences are $\mathcal{C}_{\mathrm{eq}} / 4 \pi M=0.87$ and $\mathcal{C}_{\text {pole }} / 4 \pi M=1.1$. By this time the disjoint horizon has an area $\mathcal{A} / 4 \pi M^{2}=1.1$ and a polar circumference $\mathcal{C}_{\text {pole }} / 2 \pi M=1.0$. 
In Figs. 7-9 we consider a sequence of collisions from rest between equilibrium clusters with successively smaller values of $a / M$. Figure 7 shows the evolution of spheres of radius $a=64 M$ separated by $z_{0}=256 M$. This case is essentially Newtonian. Following coalescence and violent relaxation, the two clusters achieve virial equilibrium. To monitor the approach to virial equilibrium, we computed the ratio $2 T /|W|$, where $T$ is the Newtonian kinetic energy and $W$ is the Newtonian gravitational potential energy. In equilibrium, this ratio is precisely unity. After some initial large oscillations, the ratio had fallen to 1.07 by the time we stopped the integration. As expected, violent relaxation produces equilibrium in just a few dynamical time scales.

Figure 8 shows a more relativistic encounter, with $a=9 M$ and $z_{0}=36 M$. The outcome is qualitatively similar to the Newtonian case. The virial ratio is no longer applicable when relativistic effects are important. How-

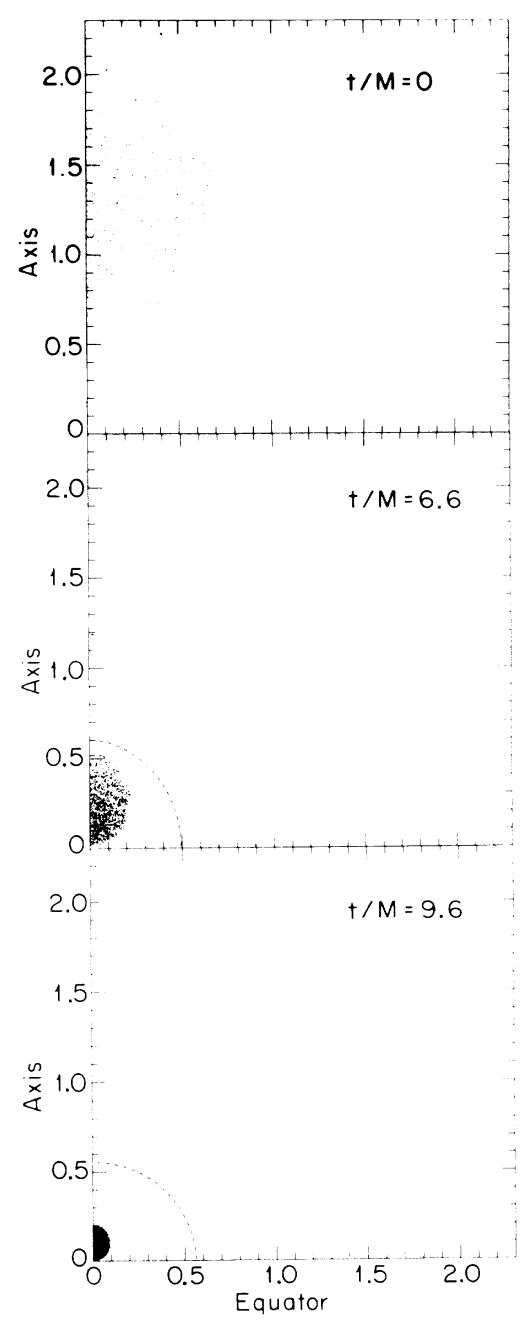

FIG. 6. Snapshots of particle positions at selected times for the collision of the same spheres shown in Fig. 3, but boosted towards each other with a velocity $v=0.5$. Now only a common horizon appears, as the spheres are in contact at the end of the integration. Its area is $\mathcal{A} / 16 \pi M^{2}=0.70$, while its circumferences are $\mathcal{C}_{\text {eq }} / 4 \pi M=0.76$ and $\mathcal{C}_{\text {pole }} / 4 \pi M=0.85$. ever, the quantity

$$
E_{0}=-\sum_{j}\left(1+u_{0}^{j}\right)
$$

becomes constant in time as the spacetime becomes stationary. Note that $E_{0}$ reduces to the total energy in the Newtonian limit. We find numerically that $E_{0}$ is con-

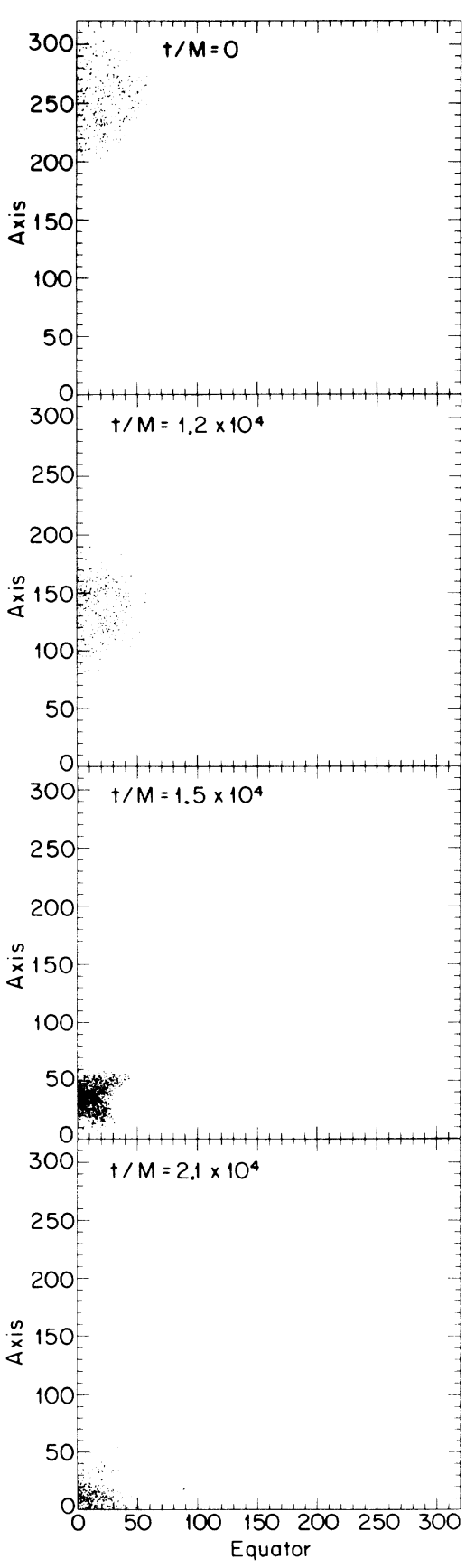

FIG. 7. Snapshots of particle positions at selected times for the collision of two equilibrium spheres of particles whose centers are initially at rest. The initial radius of each sphere is $a=64 M$, and their centers are at $z_{0}= \pm 256 M$, so that this case is essentially in the Newtonian regime. Following collision and coalescence, the spheres virialize. 
served over several orbital periods towards the end of our integrations, indicating that we have reached a steady state.

By contrast, Fig. 9 shows the evolution when $a=7 M$ and $z_{0}=28 M$. Now the merger results in collapse to a black hole. Thus the critical radius at which the transition from stable merger to catastrophic collapse occurs

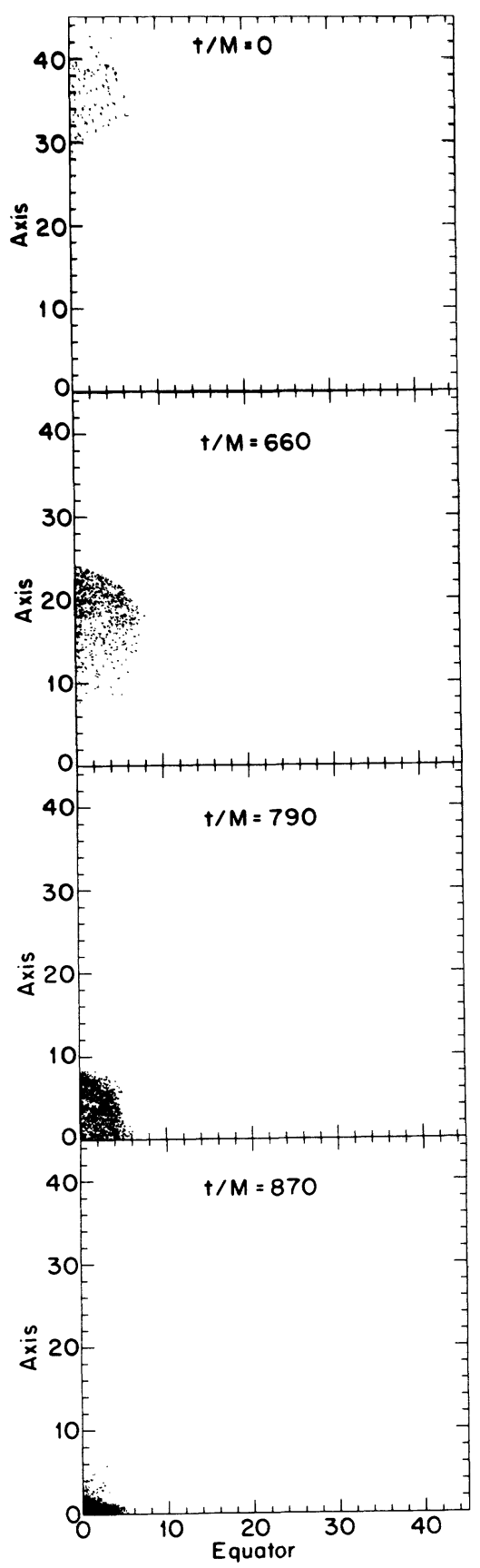

FIG. 8. Snapshots of particle positions at selected times for the collision of two equilibrium spheres of particles whose centers are initially at rest. The initial radius of each sphere is $a=9 M$, and their centers are at $z_{0}= \pm 36 M$. While this case is more relativistic than in Fig. 7 , the behavior is qualitatively the same. lies in the range $7 M \lesssim a \lesssim 9 M$.

The real significance of this numerical experiment is that we expect analogous behavior for the head-on collision of neutron stars. In particular, the collision of two neutron stars, each of which is below the maximum mass

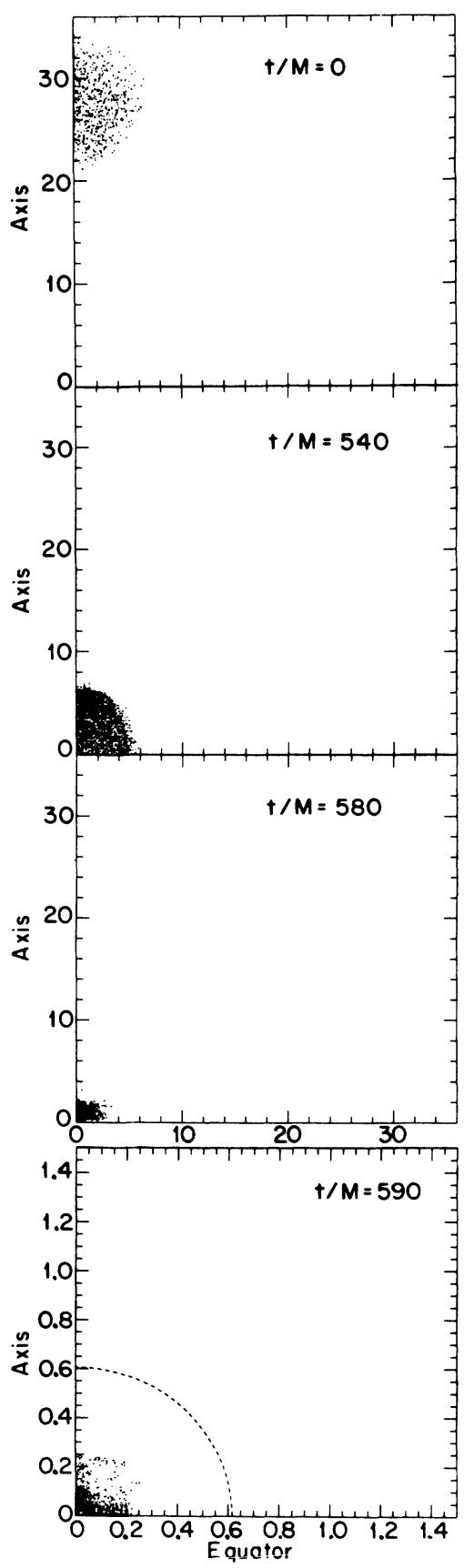

FIG. 9. Snapshots of particle positions at selected times for the collision of two equilibrium spheres of particles whose centers are initially at rest. The initial radius of each sphere is $a=7 M$, and their centers are at $z_{0}= \pm 28 M$. Now the spheres are sufficiently relativistic that the collision is followed by catastrophic collapse to a black hole. The common horizon shown in the blowup at $t / M=590$ has an area $\mathcal{A} / 16 \pi M^{2}=1$.1, while its circumferences are $\mathcal{C}_{\text {eq }} / 4 \pi M=1.1$ and $\mathcal{C}_{\text {pole }} / 4 \pi M=1.0$. 
limit, can result in a merged configuration that is too massive to be stable. The collapse may be postponed, however, if the fluid is sufficiently shock heated that thermal pressure provides an appreciable fraction of the total pressure support. This behavior has already been demonstrated for the collapse of single spherical stars above the maximum mass [15]. These stars can exist in stable equilibrium as long as their temperature remains high. Eventually, after neutrino cooling, any neutron star must collapse if its total mass exceeds the cold mass limit. For collisions between $1.4 M_{\odot}$ neutron stars, however, thermal pressure may be unable to impede dynamical collapse. Just as for cluster collisions, where there is a critical value of $a / M$, there should be a critical mass for neutron-star collisions above which collapse will occur even on a dynamical time scale.

\section{DISCUSSION}

Solving the Einstein equations with matter provides an alternative means of studying black-hole collisions. There are some crucial computational differences between this approach and solving the vacuum Einstein equations: on the one hand, one has to solve the matter evolution equations; on the other hand, one avoids ambiguities associated with the interior topology of the black holes [16]. Having constructed a code that handles matter, one can easily use it to set up initial data and evolve it for the collision of two black holes.

With only moderate computational resources, our present code can get very accurate initial data. It can also reliably evolve the data in order to assess the final fate of a collision event. In particular, it can determine whether or not colliding spheres of particles pass through each other or coalesce. In the latter case, it can determine whether the merging spheres virialize, or whether they collapse to black holes. In all cases, however, the emitted gravitational radiation is so small that we cannot determine its precise magnitude with these resources. Part of the problem is fluctuations in the matter source terms caused by the finite number of particles in the sample.
Even if one carried out a simulation with a very large number of particles and grid zones, there is an additional problem associated with the choice of coordinates. For example, for the collision depicted in Fig. 3, the black holes have hardly approached each other by the time we are forced to terminate the integration. As we discovered in our studies of spherical collapse [14], maximal time slicing with isotropic coordinates is inadequate to follow late time evolution well after a black hole forms - the central singularity grows too rapidly for numerical accuracy to be retained. In the spherical case we showed that a combination of polar time slicing and the introduction of "freezing" variables allows one to integrate far into the future. Clearly, a suitable generalization of these coordinates needs to be explored for the nonspherical case.

\section{ACKNOWLEDGMENTS}

This research was supported in part by NSF Grants Nos. AST 90-15451 and PHY 90-07834 and NASA Grant No. NAGW-2364 at Cornell University. Computations were performed on the Cornell National Supercomputer Facility.

\section{APPENDIX}

Here we list the key equations for the evolution of an axisymmetric configuration in the ADM 3+1 decomposition of general relativity. We specialize to the case of no net angular momentum [17]. We adopt the spacetime coordinates $(t, r, \theta, \phi)$. Because of axisymmetry all quantities are functions only of $(t, r, \theta)$.

The metric is given by Eq. (2.1). In addition to the metric coefficients $\alpha, \beta^{r}, \beta^{\theta}, A$, and $B$, the ADM formalism introduces the components of the extrinsic curvature tensor $K^{i}{ }_{j}$. It is convenient to introduce the related quantities

$$
\widehat{K}_{j}^{i} \equiv A^{2} B K_{j}^{i}
$$

The evolution equations, which we use to determine the "radiation variables" $\eta=\ln (A / B)$ and $\widehat{K}_{\theta}^{r}$, are

$$
\begin{aligned}
\partial_{t} \eta= & \frac{\alpha}{A^{2} B} \hat{\lambda}+\beta^{r} \partial_{r} \eta+\beta^{\theta} \partial_{\theta} \eta+\partial_{\theta} \beta^{\theta}-\beta^{\theta} \cot \theta \\
\partial_{t} \hat{K}_{\theta}^{r}= & \frac{1}{r^{2}} \partial_{r}\left(r^{2} \beta^{r} \hat{K}_{\theta}^{r}\right)+\frac{1}{\sin \theta} \partial_{\theta}\left(\sin \theta \beta^{\theta} \hat{K}_{\theta}^{r}\right)-\frac{\alpha A}{r \sin \theta}\left[\partial_{\theta}\left(\frac{\sin \theta}{A} \partial_{r}(B r)\right]-\frac{1}{A^{2}} \partial_{r}(A r) \partial_{\theta}(B \sin \theta)\right] \\
& +\hat{K}_{\theta}^{r}\left(\partial_{\theta} \beta^{\theta}-\partial_{r} \beta^{r}\right)+\left(2 \hat{\lambda}-3 \hat{K}_{\phi}^{\phi}\right) \partial_{\theta} \beta^{r}-A B \partial_{\theta}\left(\frac{1}{A} \partial_{r} \alpha\right)+\frac{B}{A r} \partial_{r}(A r) \partial_{\theta} \alpha-\frac{\alpha}{A^{2}} S_{r \theta}
\end{aligned}
$$

where

$$
\hat{\lambda}=\hat{K}_{r}^{r}+2 \hat{K}_{\phi}^{\phi}
$$

and where matter source terms such as $S_{r \theta}$ will be defined below.

The momentum constraint equations, which we use to solve for $\widehat{K}_{r}^{r}$ and $\widehat{K}_{\phi}^{\phi}$, are

$$
\frac{1}{\sin \theta} \partial_{\theta}\left(\sin \theta \hat{K}_{r}^{r}\right)+\frac{T}{\sin ^{2} \theta} \partial_{\theta}\left[\frac{\sin ^{2} \theta}{T} \hat{K}_{\phi}^{\phi}\right)=-S_{\theta}+\frac{1}{r^{2}} \partial_{r}\left(r^{2} \hat{K}_{\theta}^{r}\right) \text {, }
$$




$$
\frac{1}{r^{3}} \partial_{r}\left(r^{3} \hat{R}_{r}^{r}\right)+\hat{R}_{\phi}^{\phi} \partial_{r} \eta=S_{r}-\frac{1}{r^{2} \sin \theta} \partial_{\theta}\left(\sin \theta \hat{R}_{\theta}^{r}\right)
$$

where $T=A / B$. The Hamiltonian constraint equation determines $\psi=B^{1 / 2}$ :

$$
\begin{aligned}
& \frac{1}{r^{2}} \partial_{r}\left(r^{2} \partial_{r} \psi\right)+\frac{1}{r^{2} \sin \theta} \partial_{\theta}\left(\sin \theta \partial_{\theta} \psi\right)=-\frac{1}{4} \psi\left\{\frac{1}{r} \partial_{r}\left(r \partial_{r} \eta\right)+\frac{1}{r^{2}} \partial_{\theta}^{2} \eta\right. \\
&\left.+\frac{1}{T^{2} \psi^{8}}\left[\hat{\lambda}^{2}-3 \hat{\lambda} \hat{K}_{\phi}^{\phi}+3\left(\hat{K}_{\phi}^{\phi}\right)^{2}+\left[\frac{\hat{R}_{\phi}^{r}}{r}\right]^{2}\right]\right\}-\frac{\rho^{*}}{4 \psi}
\end{aligned}
$$

To impose the maximal slicing condition on the time coordinate, we solve the "lapse" equation for $\alpha$ :

$$
\begin{aligned}
\frac{1}{r^{2}} \partial_{r}\left[r^{2} \partial_{r}(\alpha \psi)\right]+\frac{1}{r^{2} \sin \theta} \partial_{\theta}\left[\sin \theta \partial_{\theta}(\alpha \psi)\right]=\frac{1}{4} \alpha \psi[ & -\frac{1}{r} \partial_{r}\left(r \partial_{r} \eta\right)-\frac{1}{r^{2}} \partial_{\theta}^{2} \eta \\
& \left.+\frac{7}{A^{2} B^{2}}\left\{\hat{\lambda}^{2}-3 \hat{\lambda} \hat{R}_{\phi}^{\phi}+3\left(\hat{\boldsymbol{K}}_{\phi}^{\phi}\right)^{2}+\left[\frac{\hat{\boldsymbol{R}}_{\theta}^{r}}{r}\right]^{2}\right\}+\frac{1}{B}\left(\rho^{*}+2 S\right)\right] .
\end{aligned}
$$

Imposing isotropic spatial coordinates leads to the "shift" equations for $\beta^{r}$ and $\beta^{\theta}$ :

$$
\begin{aligned}
& r \partial_{r}\left(\frac{\beta^{r}}{r}\right)-\partial_{\theta} \beta^{\theta}=\frac{\alpha}{A^{2} B}\left(2 \hat{\lambda}-3 \hat{R}_{\phi}^{\phi}\right), \\
& r \partial_{r} \beta^{\theta}+\partial_{\theta}\left(\frac{\beta^{r}}{r}\right)=\frac{2 \alpha}{A^{2} B r} \hat{R}_{\theta}^{r} .
\end{aligned}
$$

The geodesic equations of motion for the collisionless particles are

$$
\begin{aligned}
& \frac{d r}{d t}=\frac{\alpha u_{r}}{\hat{u} A^{2}}-\beta^{r}, \\
& \frac{d \theta}{d t}=\frac{\alpha u_{\theta}}{\hat{u} A^{2} r^{2}}-\beta^{\theta}, \\
& \frac{d \phi}{d t}=\frac{\alpha}{B^{2} r^{2} \sin ^{2} \theta} \frac{u_{\phi}}{\hat{u}} \text {, } \\
& \frac{d u_{r}}{d t}=-\hat{u} \partial_{r} \alpha+u_{r} \partial_{r} \beta^{r}+u_{\theta} \partial_{r} \beta^{\theta} \\
& -\frac{\alpha}{2 \hat{u}}\left[\partial_{r}\left[\frac{1}{A^{2}}\right] u_{r}{ }^{2}+\partial_{r}\left(\frac{1}{r^{2} A^{2}}\right] u_{\theta}{ }^{2}\right. \\
& \left.+\partial_{r}\left(\frac{1}{B^{2} r^{2}}\right) \frac{u_{\phi}^{2}}{\sin ^{2} \theta}\right] \\
& \frac{d u_{\theta}}{d t}=-\hat{u} \partial_{\theta} \alpha+u_{r} \partial_{\theta} \beta^{r}+u_{\theta} \partial_{\theta} \beta^{\theta} \\
& -\frac{\alpha}{2 \hat{u}}\left(\partial_{\theta}\left(\frac{1}{A^{2}}\right) u_{r}{ }^{2}+\partial_{\theta}\left(\frac{1}{r^{2} A^{2}}\right) u_{\theta}{ }^{2}\right. \\
& \left.+\partial_{\theta}\left[\frac{1}{B^{2} r^{2} \sin ^{2} \theta}\right] u_{\phi}^{2}\right] \text {, }
\end{aligned}
$$

$$
\frac{d u_{\phi}}{d t}=0
$$

where the renormalization condition $u^{\mu} u_{\mu}=-1$ gives

$\hat{u} \equiv \alpha u^{0}=\left(1+\frac{u_{r}{ }^{2}}{A^{2}}+\frac{u_{\theta}{ }^{2}}{r^{2} A^{2}}+\frac{u_{\phi}{ }^{2}}{B^{2} r^{2} \sin ^{2} \theta}\right)^{1 / 2}$.

The particles are binned to determine the source terms for the field equations:

$$
\begin{aligned}
& \rho^{*}=\sum_{j} \frac{m \hat{u}_{j}}{\left(r^{2} \sin \theta \Delta r \Delta \theta \Delta \phi\right)_{j}}, \\
& S_{r}=\sum_{j} \frac{m u_{r}^{j}}{\left(r^{2} \sin \theta \Delta r \Delta \theta \Delta \phi\right)_{j}}, \\
& S_{\theta}=\sum_{j} \frac{m u_{\theta}^{j}}{\left(r^{2} \sin \theta \Delta r \Delta \theta \Delta \phi\right)_{j}}, \\
& S_{r \theta}=\sum_{j} \frac{m u_{r}^{j} u_{\theta}^{j}}{\hat{u}_{j}\left(r^{2} \sin \theta \Delta r \Delta \theta \Delta \phi\right)_{j}}, \\
& S=\rho^{*}-\sum_{j} \frac{m}{\hat{u}_{j}\left(r^{2} \sin \theta \Delta r \Delta \theta \Delta \phi\right)_{j}} .
\end{aligned}
$$
by

The Brill mass of the spactime is given at any instant $M=\frac{1}{2 \pi} \int d^{3} x\left[(\nabla \ln \psi)^{2}+\widehat{K}_{i j} \hat{R}^{i j}\left[\frac{1}{8 A^{2} B^{2}}\right]+\frac{A^{2}}{4} \rho^{*}\right]$,

where $\nabla$ is the flat-space gradient operator. 
[1] S. L. Shapiro and S. A. Teukolsky, Phys. Rev. Lett. 66, 994 (1991).

[2] S. L. Shapiro and S. A. Teukolsky, Am. Sci. 79, 330 (1991).

[3] L. L. Smarr, in Sources of Gravitational Radiation, edited by L. L. Smarr (Cambridge University Press, Cambridge, England, 1979), p. 245.

[4] L. L. Smarr and J. R. Wilson, as reported by J. R. Wilson, in Sources of Gravitational Radiation [3], p. 443; D. L. Gilden and S. L. Shapiro, Astrophys. J. 287, 728 (1984); C. R. Evans, in Relativistic Astrophysics, Proceedings of the 13th Texas Symposium, Chicago, Illinois, 1986, edited by M. P. Ulmer (World Scientific, Singapore, 1987), p. 152; C. S. Kochanek and C. R. Evans, in Frontiers in Numerical Relativity, edited by C. R. Evans, L. S. Finn, and D. Hobill (Cambridge University Press, Cambridge, England, 1989), p. 297.

[5] See, e.g., C. S. Kochanek, S. L. Shapiro, S. A. Teukolsky, and D. F. Chernoff, Astrophys. J. 358, 81 (1990).

[6] R. Arnowitt, S. Deser, and C. W. Misner, in Gravitation: An Introduction to Current Research, edited by L. Witten (Wiley, New York, 1962), p. 227.
[7] T. Nakamura, S. L. Shapiro, and S. A. Teukolsky, Phys. Rev. D 38, 2972 (1988).

[8] C. W. Misner and J. A. Wheeler, Ann. Phys. (N.Y.) 2, 525 (1957).

[9] A. Einstein, Ann. Math. 40, 922 (1939).

[10] S. L. Shapiro and S. A. Teukolsky, Astrophys. J. 298, 34 (1985).

[11] K. Eppley, Phys. Rev. D 16, 1609 (1977).

[12] Note that one cannot use $\theta$ as the independent variable as in Ref. [7] when searching for disjoint horizons, since $\theta$, unlike $s$, is not monotonic along the curve.

[13] N. T. Bishop, Gen. Relativ. Gravit. 14, 717 (1982).

[14] S. L. Shapiro and S. A. Teukolsky, Astrophys. J 307, 575 (1986).

[15] S. L. Shapiro and S. A. Teukolsky, Astrophys. J. 235, 199 (1980).

[16] R. W. Lindquist, J. Math. Phys. 4, 938 (1963). For a recent discussion, see G. B. Cook, Phys. Rev. D 44, 2983 (1991).

[17] C. R. Evans, Ph.D. thesis, University of Texas at Austin, 1984. 